\title{
External Apical Root Resorption after Six and 12 months of Non- Extraction Orthodontic Treatment
}

\section{Paula Cabrini Scheibel ${ }^{1 *}$, Kelly Regina Micheletti ${ }^{2}$ and Adilson Luiz Ramos ${ }^{3}$}

${ }^{1}$ Orthodontist, Master's degree from the Dental School of the Universidade Estadual de Maringá, Maringá, Paraná, Brazil

${ }^{2}$ Master's Student in Integrated Dentistry, Universidade Estadual de Maringá, Maringá, Paraná, Brazil

${ }^{3}$ Orthodontist, PhD, Adjunct Professor at the Dental School of the Universidade Estadual de Maringá, Maringá, Paraná, Brazil

\begin{abstract}
Objectives: The aim of the present study was to test the hypothesis that external apical root resorption (EARR) after six months of orthodontic treatment could be an incidence indicator of EARR after 12 months of treatment in non extraction orthodontic cases. A comparison of EARR between different types of root morphology was also performed.

Material and Methods: Periapical radiographs of the upper incisors were obtained prior to treatment $\left(\mathrm{T}_{1}\right)$ as well as at six months $\left(T_{2}\right)$ and 12 months $\left(T_{3}\right)$ of non-extraction orthodontic treatment among 47 patients aged 11 years or older. The roots were classified based on anatomic shape. Triangular, pipette-shaped, bent and/or short roots were classified as having a tendency toward EARR, whereas those with a rhomboidal and rectangular shape were classified as having no tendency toward EARR.
\end{abstract}

Results: At 12 months of orthodontic treatment EARR ranged from 0 to $12.1 \%$ of total tooth length (mean: $3.5 \%$; SD: 3.03), which meant 0 to $2.7 \mathrm{~mm}$ of EARR. There was significant correlation between EARR at six months and EARR at 12 months $(r=0.7606 ; p<0.0001)$. There was no correlation between root shape and EARR.

Conclusions: EARR after the first six months of orthodontic treatment was a good incidence indicator of EARR after 12 months of treatment $(r=0.8)$. Root shape did not show significant influence in root resorption level in non extraction orthodontic cases.

Keywords: Orthodontic tooth movement; Root resorption; Periapical radiographs

\section{Introduction}

One of the challenges of orthodontics is the management of external apical root resorption (EARR), which is a common undesirable consequence of orthodontic treatment. Although root resorption does not substantially compromise root integrity in most patients, it is severe in 5\% to $14.5 \%$ and can compromise dental support in such cases [1-6]

Many factors have been reported to influence the degree of EARR during orthodontic treatment, but there is no consensus on predictive factors of this condition. The following have been reported as possible agents involved in EARR: tooth morphology; morphology and density of the alveolar bone; crown-root proportion; aspects of orthodontic mechanics, such as frequency and magnitude of the force, extension and type of dental movement; history of dental trauma; and genetic factors, such as the presence of the P2X7 receptor [3-13]. There are few studies on the predictive power that EARR recorded in the early stages of treatment may have on subsequent phases [14-16]. Moreover, the relationship between EARR and root shape remains controversial. A number of studies reports a significant association between root resorption and different types of root morphology, such as pipetteshaped, narrow or bent [2-17]. However, other studies have not confirmed this association $\left[{ }^{3}-18\right]$. Considering the limited effect of risk factors identified for EARR during active orthodontic treatment, studies involving multivariate analysis suggest that individual predisposition could be the main etiological factor [1-16]. Up until as we know, there is also no specific study with a sample comprised only of non extraction orthodontic cases, once it has been reported that cases involving premolar extractions are more subject to greater degrees of EARR [6-22].

The aim of the present study was to test the hypothesis that EARR after six months of orthodontic treatment may give an indication of the incidence of some EARR after 12 months of treatment in non extraction orthodontic cases. A comparison of EARR between different types of root morphology was also performed.

\section{Materials and Methods}

The sample in the present prospective study was made up of 91 upper central incisors of 47 patients aged 11 years and older, who had their complete fixed orthodontic appliance installed (straightwire technique) by orthodontic graduate students from July 2008 to April 2009. Signed informed consent was the primary condition for the inclusion of each patient. The following were the other inclusion criteria: no past history of fixed orthodontic treatment; no past history of dentoalveolar trauma in the region of the upper incisors; upper incisors with either intact crown or only proximal restorations; nonextraction orthodontic treatment plan. The study received approval from the Ethics Committee on Research Involving Human Subjects of the Universidade Estadual de Maringá (Brazil) (\#190/2008).

Periapical radiographs were taken of the upper incisor region in each participant on three occasions: initial ( $\mathrm{T}_{1}$, immediately prior to or immediately after placement of the braces), at six months $\left(\mathrm{T}_{2}\right)$ and 12 months $\left(\mathrm{T}_{3}\right)$ of orthodontic treatment. The radiographic equipment used was either the Pro 70-Intra (Prodental, RibeirãoPreto, São Paulo, Brazil) or RX Timex $70 \mathrm{Col}$ (Gnatus, RibeirãoPreto, São Paulo,

*Corresponding author: Dr. Paula Cabrini Scheibel, Dental office, Av. Luiz Teixeira, 2266, CEP: 87010-370, Maringá, Paraná, Brazil, Tel: (05544) 30262613; E-mail: paulascheibel@msn.com

Received July 20, 2011; Accepted August 12, 2011; Published August 23, 2011

Citation: Scheibel PC, Micheletti KR, Ramos AL (2011) External Apical Root Resorption after Six and 12 months of Non-Extraction Orthodontic Treatment. Dentistry 1:102. doi:10.4172/2161-1122.1000102

Copyright: () Scheibel PC, et al. This is an open-access article distributed under the terms of the Creative Commons Attribution License, which permits unrestricted use, distribution, and reproduction in any medium, provided the original author and source are credited. 
Brazil), with a 0.25 -second exposure time. The film was processed in a standardized time/temperature method. The images were then digitalized on a scanner (ArtixScan 18000F, Microtek) with a resolution of 400 ppi for subsequent computerized measurement of the amount of apical root resorption (CorelDRAW X4 program).

\section{Radiographic analysis}

Measurement of external apical root resorption: The length of the upper central incisors (teeth 11 and 12) and respective crowns was measured on the three occasions $\left(\mathrm{DT}_{1}, \mathrm{DT}_{2}, \mathrm{DT}_{3}\right.$ and $\mathrm{CT}_{1}, \mathrm{CT}_{2}, \mathrm{CT}_{3}$, respectively) to a precision of $0.1 \mathrm{~mm}$ with the aid of the CorelDRAW X4 program $[23,24]$. These measurements respectively corresponded to the distance from the incisal border to the root apex and the greatest distance between the incisal border and cementum-enamel junction, using the long axis of the tooth as reference (Figure 1). In order to compensate for possible variations in the inclination of the radiographic takes on the different occasions, supposing that the crown measurement remains unaltered throughout treatment, the expected tooth length at $\mathrm{T}_{2}$ (expected $\mathrm{DT}_{2}$ ) was calculated using the following equation $[23,24]$ : expected $\mathbf{D T}_{2}=\left(\mathrm{CT}_{2} \cdot \mathrm{DT}_{1}\right) / \mathrm{CT}_{1}$. The amount of EARR was determined by subtracting the expected tooth length at $\mathrm{T}_{2}$ from the tooth length measured at $\mathrm{T}_{2}$ : EARR at $\mathrm{T}_{2}=\operatorname{expected} \mathrm{DT}_{2}-$ $\mathrm{DT}_{2}$. The same procedure was used to determine EARR at $\mathrm{T}_{3}$.

The amount of root resorption was calculated in millimeters and then expressed in percentage values in relation to the initial tooth size. Teeth with resorption percentage of zero were classified as having undergone no resorption, whereas those with 1 to $4 \%$ resorption were classified as having apical rounding. Resorption between $4 \%$ and $8 \%$ was considered mild and resportion between $8 \%$ and $12 \%$ was considered moderate.

\section{Determination of root morphology}

Levander and Malmgren [14] classified roots as normal, short, blunt, with apical bent and pipette shape, and Consolaro [5] classified roots as triangular, rhomboid and rectangular based on the shape of the apical third. These root anatomies are considered in the judgment of the susceptibility to apical resorption (morphologic risk). Thus, the roots in the initial radiographic images were classified as (1) with a tendency toward EARR and (2) without a tendency toward EARR. Triangular,

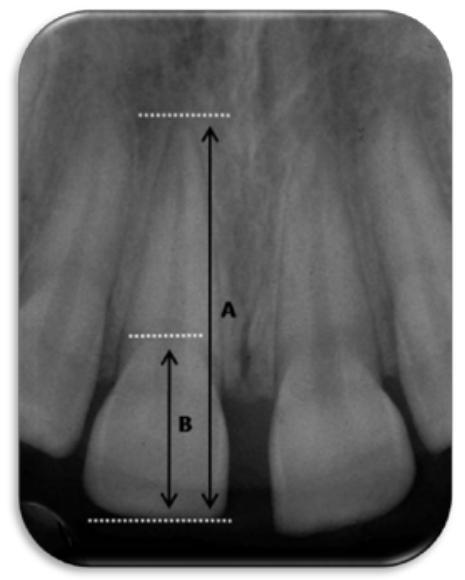

Figure 1: Radiography illustrating the tooth measurement method used; (A) incisal-apical distance (tooth length) used to calculate root resorption; (B) distance from incisal border to cementum-enamel junction (crown length) used for correction of radiographic shortening or lengthening.

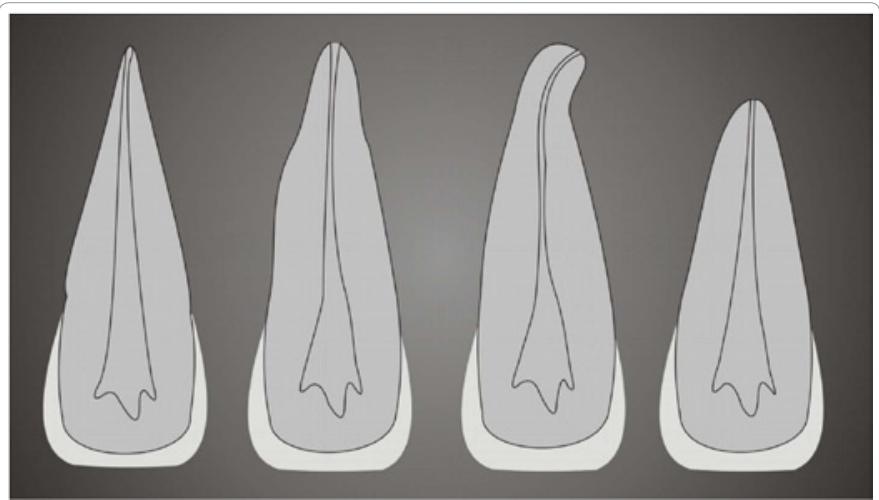

Figure 2: Root shapes with tendency toward EARR; (A) triangular root; (B) root with apical pipette shape; (C) root with apical bent; (D) short root (based on Levander and Malmgren ${ }^{14}$ and Consolaro ${ }^{5}$ classification).

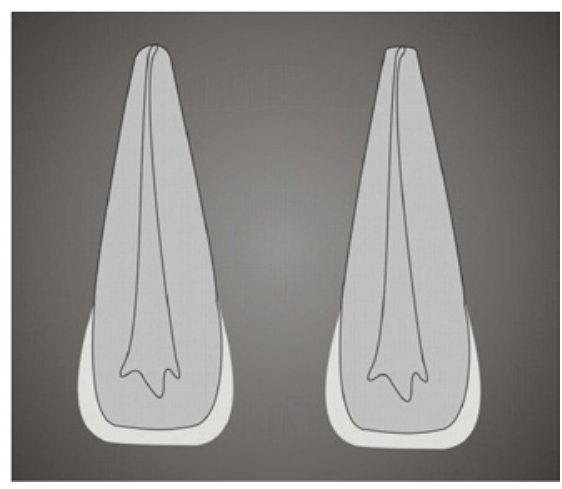

Figure 3: Root shapes without tendency toward EARR; (A) root with apical rhomboid shape; (B) root with apical rectangular shape (based on Levander and Malmgren ${ }^{14}$ and Consolaro ${ }^{5}$ classification).

pipette shape, apical bent and short roots were grouped as with a tendency toward EARR (Figure 2) and rhomboid and rectangular roots were grouped as without a tendency toward EARR (Figure 3).

Two examiners classified root morphology $($ Kappa $=0.96)$. A consensus was obtained in cases of divergence. Intra-examiner reliability regarding EARR was statistically analyzed by the difference between duplicate measurements on the radiographic images of 25 randomly selected patients at $\mathrm{T}_{1}, \mathrm{~T}_{2}$ and $\mathrm{T}_{3}$, with a two-week interval between assessments. The error of the method was calculated using Dahlberg's formula:

$$
S e=\sqrt{\frac{\sum d^{2}}{2 n}}
$$

in which $d$ is the difference between pairs of measurements and $n$ is the number of pairs of measurements [25]. Spearman's correlation coefficient ( $r$ ) was also employed. Although there were no statistically significant differences between the first and second measurements, the mean of each region measured was used in the subsequent statistical texts in order to minimize the random error.

Examiner took tooth measurements without knowing group identification. Also professionals who treated sample patients did not know study groups. 


\section{Statistical analysis}

EARR at $\mathrm{T}_{2}$ and $\mathrm{T}_{3}$ did not exhibit normal distribution (Lilliefors test). Therefore, the non-parametric Spearman correlation test was performed. The Mann-Whitney test was used for the comparison of the amount of EARR between groups with and without a tendency toward resorption. Simple linear regression analysis was used to formulate an equation for estimating the amount of resorption after 12 months in relation to resorption after six months of treatment. The level of significance was set at $5 \%$ for all statistical tests.

\section{Results}

There were no significant differences in EARR between incisors 11 and 21. Root resorption of the upper central incisors after six months of treatment (EARR $\mathrm{T}_{2}$ ) ranged from 0 to $10.7 \%$ (mean: $2.1 \%$; $\mathrm{SD}$ : 2.38) (Table 1). No root resorption occurred in ten patients $(21 \%)$ in this period. Considering the more resorbed incisor, 18 patients $(38 \%)$ exhibited 1 to $4 \%$ resorption (rounded apex); 18 (38\%) exhibited between 4 and $8 \%$ resorption (mild) and one patient (2\%) exhibited between 8 and 12\% resorption (moderate).

After 12 months of treatment, EARR ranged from 0 to $12.1 \%$ (mean: 3.5\%; SD: 3.03 ) (Table 1). Three patients (6\%) had no resorption in this period. Eighteen patients (38\%) exhibited 1 to $4 \%$ resorption (rounded apex); 18 (38\%) exhibited between 4 and $8 \%$ resorption (mild) and eight patients (17\%) exhibited between 8 and $12 \%$ resorption (moderate) (Table 2).

There were no significant differences in EARR between the groups of roots with and without a tendency toward resorption (morphologic

\begin{tabular}{|l|c|c|c|c|}
\hline & Minimum & Maximum & Mean & SD \\
\hline Age (years) & 11 & 51 & 20 & 10.52 \\
\hline EARR T $(\%)$ & 0.0 & 10.7 & 2.1 & 2.38 \\
\hline EARR T $_{3}(\%)$ & 0.0 & 12.1 & 3.5 & 3.03 \\
\hline
\end{tabular}

Table 1: Descriptive statistics of the sample $(n=47)$ in relation to age and EARR (\%) after six $\left(T_{2}\right)$ and $12\left(T_{3}\right)$ months of treatment on 91 upper central incisors.

\begin{tabular}{|c|c|c|}
\hline & \multicolumn{2}{|c|}{ Patients $\mathrm{n}(\%)$} \\
\hline EARR $\%$ & $\mathrm{~T}_{2}$ & $\mathrm{~T}_{3}$ \\
\hline 0 & $10(21 \%)$ & $3(6 \%)$ \\
\hline$>1$ and $\leq 4$ & $18(38 \%)$ & $18(38 \%)$ \\
\hline$>4$ and $\leq 8$ & $18(38 \%)$ & $18(38 \%)$ \\
\hline Tond $\leq 12$ & $1(2 \%)$ & $8(17 \%)$ \\
\hline Total & $47(100 \%)$ & $47(100 \%)$ \\
\hline
\end{tabular}

Table 2: Descriptive statistics of the number of patients in relation to percentage of root resorption after six $\left(\mathrm{T}_{2}\right)$ and $12\left(\mathrm{~T}_{3}\right)$ months of treatment (more resorbed central incisor).

\begin{tabular}{|l|c|c|c|c|c|}
\hline & \multicolumn{2}{|c|}{ With tendency } & \multicolumn{2}{c|}{ Without tendency } & P \\
\hline & \multicolumn{2}{|c|}{$(26 / 91)$} & \multicolumn{2}{|c|}{$(65 / 91)$} & \\
\hline & Mean (\%) & SD & Mean (\%) & SD & \\
\hline EARR T $_{2}$ & 2.07 & 3.047 & 2.09 & 2.152 & $0.151 \mathrm{~ns}$ \\
\hline EARR T & 2.99 & 3.583 & 3.70 & 2.755 & $0.079 \mathrm{~ns}$ \\
\hline
\end{tabular}

Table 3: Comparison of EARR (\%) at $\mathrm{T}_{2}$ and $\mathrm{T}_{3}$ between groups with and without tendency toward root resorption (Mann-Whitney test).

\begin{tabular}{|c|c|c|c|c|c|}
\hline \multicolumn{3}{|c|}{ TMWith tendency } & \multicolumn{2}{c|}{ Without tendency } \\
\hline Triangular & Pipette-shaped & Bent & Short & Rhomboidal & Rectangular \\
\hline $11(42,3 \%)$ & $11(42,3 \%)$ & $3(11,5 \%)$ & $6(23,1 \%)$ & $62(\%)$ & $3(\%)$ \\
\hline \multicolumn{3}{|c|}{$26(28,6 \%)$} & $65(71,4 \%)$ \\
\hline
\end{tabular}

Table 4: Descriptive statistics of the evaluated upper central incisors $(n=91)$ in relation to determination of root morphology.

risk) after either six $(P=0.151)$ or $12(P=0.079)$ months of treatment. Among the 91 incisors analyzed, 26 had root morphology with a tendency toward EARR and 65 had root morphology without a tendency toward EARR (Table 3).

Descriptive statistics of the evaluated upper central incisors $(\mathrm{n}=91)$ in relation to determination of root morphology are presented in Table 4.

There was a significant correlation between EARR at $\mathrm{T}_{2}$ and at $\mathrm{T}_{3}$ $(\mathrm{r}=0.7606 ; P=0.000)$. The simple linear regression analysis revealed that the amount of EARR at $\mathrm{T}_{2}$ was associated to EARR at $\mathrm{T}_{3}\left(\mathrm{r}^{2}=0.64\right.$, $P=0.000)$. The percentage of EARR at $\mathrm{T}_{3}$ was estimated by the following formulas: EARR $\mathrm{T}_{3}=1.436+0.9957\left(\mathrm{EARR}_{2}\right)$.

Figures 4 and 5 display the scatter plot regarding RRE after 6 and 12 months of treatment in millimeters and percentage, respectively.

\section{Discussion}

Previous reports have suggested a correlation between root morphology and EARR during orthodontic movement, with pipette shape, triangular and bent roots associated to resorption [2-17]. The likely explanation for the greater tendency toward EARR is the possibility of a greater concentration of forces in thinner apical root shapes and, consequently, greater harm to the cementum [5]. While this may be a plausible reason, the association between the dissipation of apical force and EARR remains unclear. Contrary to previously raised hypothesis, there were no statistically significant differences in the present study regarding the percentage of root resorption between root groups with and without a supposed tendency toward EARR (Table 3). A number of recent studies has also reported a weak or no correlation between root shape and EARR [3-18]. Moreover, Smale [4] report that, while narrow and bent roots may exhibit an increased risk of EARR in the early stages of treatment, the explanation of the variance of these risk factors is less than $25 \%$.

The literature is also divergent with regard to the greater or lesser probability of EARR in short roots. Taithongchai [2] found a significant association between EARR and root size, with short roots exhibiting a greater tendency toward resorption, although the EARR was not clinically significant. However, a number of studies, including the present investigation, have found that short roots do not constitute a risk factor for EARR [1-18].

In the present study, the amount of root resorption was assessed in millimeters and then expressed in percentage. From the clinical standpoint, even mild resorption in short roots may be more important than the same absolute amount in long roots. Thus, although short roots may not be more prone to resorption, care must be taken with this particular root shape. For example, the incisor \#38 had a $27 \mathrm{~mm}$ total length before treatment and after 12 months it presented $2.0 \mathrm{~mm}$ of EARR, which meant $7.3 \%$ of tooth reduction. While a shorter incisor (\#42) with $18.7 \mathrm{~mm}$ total length prior to orthodontic treatment presented after 12 months almost the same level of EARR 
$(2.1 \mathrm{~mm})$, but reflected in $11.2 \%$ of tooth reduction. Furthermore, it must be noted that if root length only is considered, instead of total dental length, percentage of reduction almost doubles, more affecting alveolar insertion. Prognosis of tooth depends upon the surface area of periodontal ligament attachment not necessarily root length and the apical surface corresponds to the smallest part of periodontal support [26]. Root apex loss of $3 \mathrm{~mm}$ equals alveolar crest bone loss of $1 \mathrm{~mm}$ from cervical margin in a normal tooth [27].

It has been reported that cases involving premolar extractions are more subject to greater degrees of EARR [6-22]. In a retrospective study of 1049 cases, Marques [6] found a high prevalence of EARR (14.5\%) at the end of treatment, with an odds ratio of 6.38 for cases treated with the extraction of first premolars. It is suggested that the greater apical movement in cases of extraction, especially in the anteroposterior direction, is the real risk factor for EARR [1-22]. According to some authors, this may be related to the approximation of the roots of the maxillary incisors to the palatine cortical bone, which has greater density, thereby providing a greater concentration of force at the apical region and possibly greater EARR [7-9]. In the present sample, no extraction cases only were included. Furthermore, the 12-month duration of the study can explain the low degree of EARR (0 to $12 \%$ or 0 to $2.7 \mathrm{~mm}$ ).

It has been reported that patients with resorption greater than 1 $\mathrm{mm}$ in the first six months of orthodontic treatment have a three times risk of severe resorption (greater than $5 \mathrm{~mm}$ ) at the end of treatment. Those with more than $2 \mathrm{~mm}$ resorption at six months have a 15 times greater chance of exhibiting severe resorption at the end of treatment ${ }^{16}$. In the present study, 18 patients $(38 \%)$ had at least one incisor with mild EARR ( $>4$ and $\leq 8 \%$ or $>1$ and $\leq 1.7 \mathrm{~mm}$ ) and one patient had moderate EARR ( $>8$ and $\leq 12 \%$ or $>1.7$ and $\leq 2.7 \mathrm{~mm}$ ) after six months of treatment. At 12 months, however, $45 \%$ of the patients had either no resorption or only apical rounding, 38\% had mild EARR and $17 \%$ exhibited moderate resorption (Table 2). This corroborates the findings of most studies that the risk of severe resorption is generally low, and confirms EARR progression during treatment [1-16].

As risk factors of EARR are not adequate predictive factors, a number of authors have sought to identify patients in the early stages of orthodontic treatment who will exhibit severe EARR at the end of treatment [14-16]. Årtun [16] found a significant correlation between EARR at six and twelve months with EARR at the end of active treatment, reporting that EARR at six months explained $46 \%$ $\left(P<0.001 ; \mathrm{r}^{2}=0.46\right)$ of EARR at the end of treatment and EARR at 12 months explained 64\% $\left(P<0.001 ; \mathrm{r}^{2}=0.64\right)$ [16]. The regression formula in the present sample explained $64 \%$ of the cases of resorption at 12 months (Figures 4 and 5). The data corroborate the significant incidence indicator power that periapical radiography after six months of treatment can have regarding the risk of EARR.

When mild to moderate EARR is detected in the six-month periapical radiograph, treatment should be halted for two to three months with passive archwires [14-28]. Halting treatment for three months in one arch while working on the other is a practical solution that can be implemented without changing the treatment plan. This protocol seems to minimize EARR progression [5-28]. However, if severe resorption is identified, the treatment goals should be reassessed with the patient, changing to alternative options as prosthetic solutions to close spaces, releasing teeth from active arches if possible and early fixation of resorbed teeth [1-18].

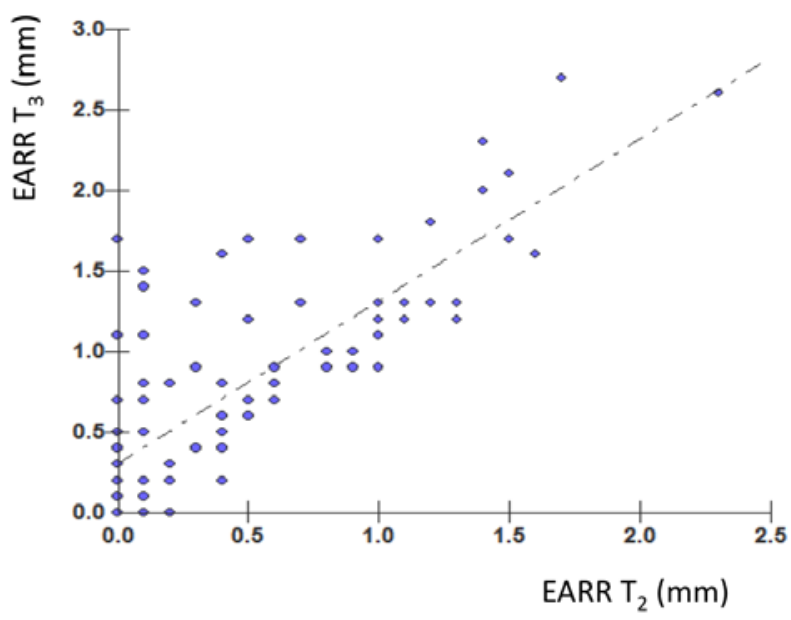

Figure 4: Scatter plot (linear regression) regarding absolute EARR in millimeters after 6 and 12 months of treatment.

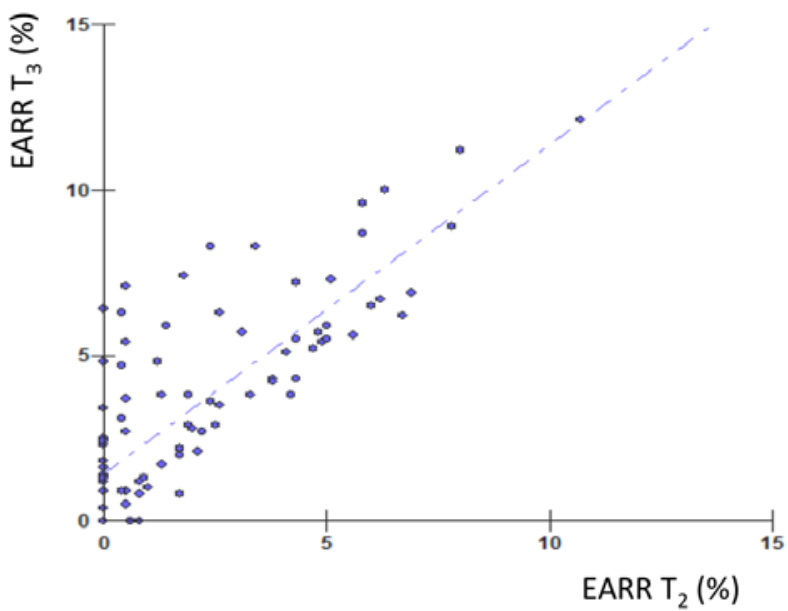

Figure 5: Scatter plot (linear regression) regarding percentage of EARR after 6 and 12 months of treatment.

\section{Conclusion}

The amount of EARR after the first six months of orthodontic treatment does give an indication of the incidence of some external apical root resorption 6 months into treatment, but could still vary in this sample depending on the internal (genetic) and external (mechanics) present in this sample.

In non extraction orthodontic cases, no significant difference was found in the amount of root resorption between roots classified as having a tendency toward EARR (triangular, bent, pipette shape and short) and those classified as not having this morphologic risk (rhomboid and rectangular).

\section{References}

1. Mirabella AD, Årtun J (1995) Risk factors for apical root resorption of maxillary anterior teeth in adult orthodontic patients. American Journal of Orthodontics and Dentofacial Orthopedics 108: 4-55.

2. Taithongchai R, Sookkorn K, Killiany DM (1996) Facial and dentoalveolar structure of apical root shortening and the prediction of apical root shortening. American Journal of Orthodontics and Dentofacial Orthopedics 110: 296 -302. 
Citation: Scheibel PC, Micheletti KR, Ramos AL (2011) External Apical Root Resorption after Six and 12 months of Non-Extraction Orthodontic Treatment. Dentistry 1:102. doi:10.4172/2161-1122.1000102

Page 5 of 5

3. Sameshima G T, Sinclair P M (2001) Predicting and preventing root resorption: Part I. Diagnostic factors. American Journal of Orthodontics and Dentofacial Orthopedics 119: 505-510.

4. Smale I, Artun J, Behbehani F, Doppel D, et al. (2005) apical root resorption 6 months after initiation of fixed orthodontic appliance therapy. American Journal of Orthodontics and Dentofacial Orthopedics 128: 57-67.

5. Consolaro A (2005) Reabsorções dentárias na movimentação ortodôntica. In: Consolaro A (ed) Reabsorções Dentárias nas especialidades clínicas. 2nd ed. Maringá: Dental Press pp. 353-400.

6. Marques LS, Ramos-Jorge ML, Rey AC, Armond MC, Ruellase ACO (2010) Severe root resorption in orthodontic patients treated with the edgewise method: Prevalence and predictive factors. American Journal of Orthodontics and Dentofacial Orthopedics 13: $384-388$

7. Kaley J, Philips C (1991) Factors related to root resorption in edgewise practice . Angle Orthodontist 6: 125-132.

8. Linge L, Linge BO (1991) Patient characteristics and treatment variables associated with apical root resorption during orthodontic treatment. American Journal of Orthodontics and Dentofacial Orthopedics 99: 35-43.

9. Horiuchi A, Hotokezaka H, Kobayashi K (1998) Correlation between cortical plate proximity and apical root resorption. American Journal of Orthodontics and Dentofacial Orthopedics 114: 311-318.

10. Segal GR, Schiffman PH, Tuncay OC (2004) Meta analysis of the treatmentrelated factors of external apical root resorption. Orthodontics and Craniofacial Research 7: 71-78.

11. Ramanathan C, Hofman Z (2009) Root resorption during orthodontic tooth movements. European Journal of Orthodontics 31: $578-583$.

12. Viecilli R F, Chen J, Katona T R, Roberts WE (2009) Comparison of Dentoalveolar Morphology in WT and P2X7R KO Mice for the Development of Biomechanical Orthodontic Models. The Anatomical Record 292: 292-298.

13. Viecilli RF, Chen J, Katona TR, Hartsfield JK, Roberts WE (2009) Orthodonticmechanotransduction and the role of the $\mathrm{P} 2 \mathrm{X} 7$ receptor. American Journal of Orthodontics and Dentofacial Orthopedics 135 (6): 694.e1-694.e16.

14. Levander E, Malmgren O (1988) Evaluation of the risk of root resorption during orthodontic treatment: A study of upper incisors. European Journal of Orthodontics 10: 30-38.

15. Årtun J, Smale I, Behbehani F, Doppel D, Van't Hof M, et al (2005) Apical Root
Resorption Six and 12 Months After Initiation of Fixed Orthodontic Appliance Therapy. Angle Orthodontist 75: 919 -926.

16. Årtun J, Van't Hullenaar R, Doppel D, Kuijpers-Jagtman AM (2009) Identification of orthodontic patients at risk of severe apical root resorption. American Journal of Orthodontics and Dentofacial Orthopedics 135: 448 -455.

17. Mohandesan $\mathrm{H}$, Ravanmehr $\mathrm{H}$, Valei $\mathrm{N}$ (2007) A radiographic analysis of external apical root resorption of maxillary incisors during active orthodontic treatment. European Journal of Orthodontics 29: 134-139.

18. Walker S (2010) Root resorption during orthodontic treatment. Evidence-Based Dentistry 11: 88 .

19. Baumrind S, Korn EL, Boyd R L (1996) Apical root resorption in othodontically treated adults. American Journal of Orthodontics and Dentofacial Orthopedics 110: $311-320$

20. Harris EF (2000) Root Resorption During Orthodontic Therapy. Seminars in Orthodontics 6: 18 -194.

21. Mcnab S, Battistutta D, Taverne A, Symons A L (2000) External apical root resorption following orthodontic treatment. Angle Orthodontist 70: $227-232$.

22. de Freitas MR, Beltrão R T, Janson G , Henriques J F, Chiqueto K (2007) Evaluation of root resorption after open bite treatment with and without extractions. American Journal of Orthodontics and Dentofacial Orthopedics 132 : 143.e15 - 143.e22

23. Spurrier SW, Hall S H , Joondeph D R, Shapiro P A, Riedel R A (1990) A comparison of apical root resorption during orthodontic treatment in endodontically treated and vital teeth. American Journal of Orthodontics and Dentofacial Orthopedics 97: 130-134

24. Esteves T, Ramos AL, Pereira C M, Hidalgo M M (2007) Orthodontic Roo Resorption of Endodontically Treated Teeth. Journal of Endodontics 33: 119 $-122$.

25. Dahlberg G (1940) Statistical methods for medical and biological students. London: George Allen and Unwin Ltd, pp. $122-132$.

26. Phillips J R (1955) Apical root resorption under orthodontic therapy. Angle Orthodontist 25: 1-22.

27. Kalkwarf K L, Krejci R F, Pao Y C (1986) Effect of apical root resorption on periodontal support. Journal of Prosthetic Dentistry 56: 317-319.

28. Brezniak N, Wasserstein A (2002) Orthodontically induced inflammatory roo resorption. Part II: The clinical aspects. Angle Orthodontist 72: 180-184 